\title{
Multicolour photometry of pulsating stars in the Galactic Bulge fields
}

\author{
Przemysław Bruś and Zbigniew Kołaczkowski \\ Instytut Astronomiczny Uniwersytetu Wrocławskiego \\ Kopernika 11, 51-622 Wrocław, Poland \\ email: brus@astro.uni.wroc.pl
}

\begin{abstract}
We present a study of photometric properties of very crowded stellar fields toward the Galactic Bulge. We performed a search for pulsating stars among thousands of variable stars from the OGLE-II survey supplementing the variability study with photometric measurements in four Johnson-Cousins $U B V I_{\mathrm{C}}$ passbands. Using these data, we analysed the properties of objects located at different distances and, whenever possible, classified them.
\end{abstract}

Keywords. Galactic Bulge, pulsating stars, extinction

\section{Archival data and follow-up observations}

The main source of data used in this work is the entire OGLE-II DIA time-series $I_{\mathrm{C}}$ band photometric database of Galactic Bulge fields (Szymański 2005). All pulsating stars described here were discovered during our multiperiodicity search in the OGLE-II database. Most of them are not present in the catalog published by the OGLE Team (Woźniak et al. 2002) because of very small amplitudes (a few mmag).

The second part of our project is based on follow-up single-epoch observations of 25 selected fields carried out with the CTIO 1-m telescope in May and June 2007. Using the Y4kCam detector $\left(20^{\prime} \times 20^{\prime}\right.$ field of view $)$, we covered a relatively small part of the OGLE-II fields in four passbands: $U$ (exposures: $1500 \mathrm{~s}), B(400 \mathrm{~s}), V(250 \mathrm{~s}), I_{\mathrm{C}}$ $(120 \mathrm{~s})$. In each field we performed profile photometry by means of the DAOPHOT-II package (Stetson 1987). To perform the transformation of our photometry to the standard system, we carried out additional observations of a nearby standard field BWC (Paczyński et al. 1999) on a photometric night of June 3/4, 2007. Accurate astrometry carried out by means of the UCAC3 catalog (Zacharias et al. 2010) allowed us to perform reliable crossidentifications with the OGLE databases. Our standardized $V$ and $I_{\mathrm{C}}$ measurements are in very good agreement with the OGLE-III photometry published by Szymański et al. (2011).

\section{Analysis and results}

In order to remove long-term and seasonal trends, we applied spline function fits as the first step of the time-series analysis. Next, we performed frequency analysis by means of the Fourier periodogram in the range between 0 and $40 \mathrm{~d}^{-1}$. A given star was selected as a variable if the signal-to-noise ratio $(\mathrm{S} / \mathrm{N})$ of the dominant frequency exceeded a detection level equal to 5 . For all stars selected through the $\mathrm{S} / \mathrm{N}$ criterion, we applied a semi-automatic search for additional frequencies by means of consecutive prewhitening and repeated Fourier analysis of the residual light curve. In each iteration all parameters of the fit were updated. As a result, we found several hundreds of multiperiodic variables in each OGLE-II field. They are good candidates for pulsators, mainly those on the mainsequence. Moreover, we divided these stars into two groups: short-period pulsators with 


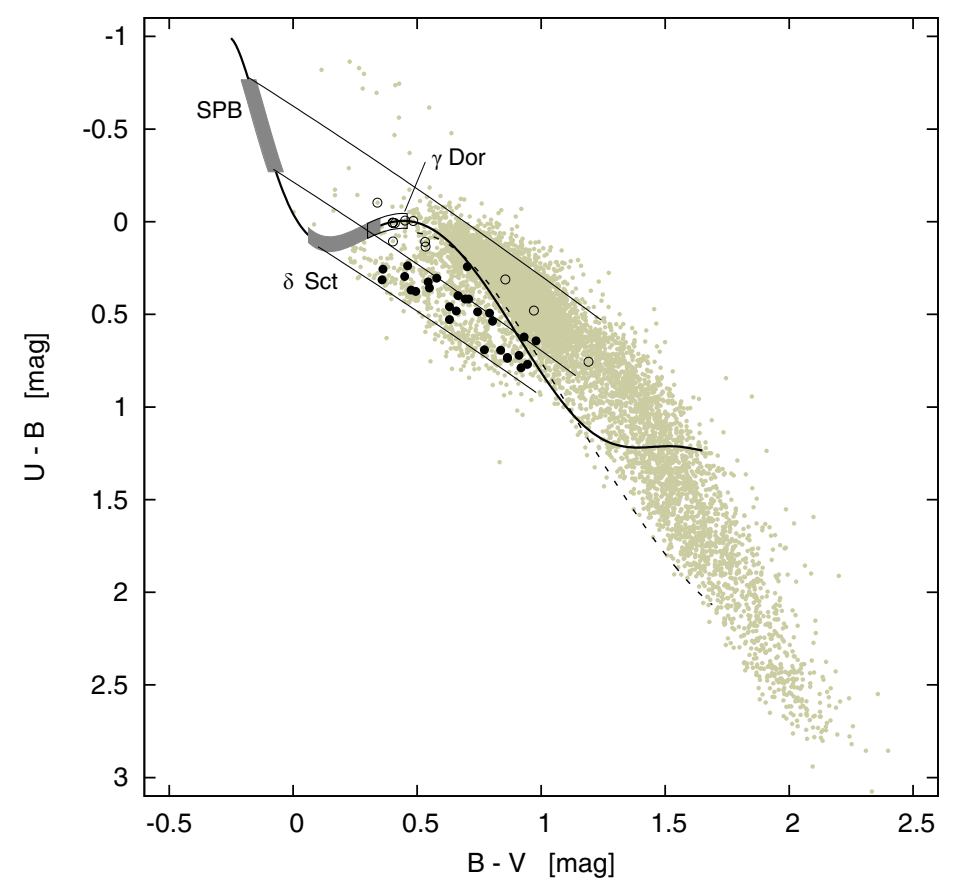

Figure 1. Observed $(U-B)$ vs. $(B-V)$ colour-colour diagram for stars in three selected fields toward the Galactic Bulge. All stars with $(U-B)$ errors smaller than 0.05 mag are shown as gray dots. The intrinsic relations for dwarfs and giants are plotted as solid and dashed curves, respectively. The schematic locations of SPB, $\delta$ Sct and $\gamma$ Dor type pulsators are marked and labeled. Stars classified as $\delta$ Sct-type variables are shown as black filled dots, SPB or $\gamma$ Dor variables as open circles. Three solid black lines indicate reddening line with a slope of 0.85 .

dominant periods shorter than $0.3 \mathrm{~d}$ and long-period pulsators including the remaining multiperiodic variables. In the first group we expect $\beta$ Cephei and $\delta$ Scuti-type stars; the second group consists of slowly pulsating B-type stars (SPB) and $\gamma$ Doradus-type stars.

The preliminary multi-colour photometry of three selected fields allowed us to make use of colour-magnitude and colour-colour diagrams in our classification of variable stars. We found pulsating stars in a wide range of colour indices measured with an accuracy better than $0.05 \mathrm{mag}$ (Fig. 1). The distribution of early A-type stars in the $(U-B)$ vs. $(B-V)$ diagram implies a significantly higher slope of the reddening line than the standard value. The whole sample of short-period variables turned out to contain only $\delta$ Scuti-type stars with $E(B-V)$ reddenings between 0.3 and 1 mag. The majority of the long-period pulsators cannot be unambiguously classified: they can be either highly reddened SPB or $\gamma$ Doradus stars.

Acknowledgments. This work was supported by the National Science Center (NCN) grant No. 2011/03/B/ST9/02667.

\section{References}

Paczyński, B., Udalski, A., Szymański, M., et al. 1999, AcA, 49, 319

Stetson, P. B. 1987, PASP, 99, 191

Szymański, M. K. 2005, AcA, 55, 43

Szymański, M. K., Udalski A., Soszyński, I., et al. 2011, AcA, 61, 83

Woźniak, P. R., Udalski, A., Szymański, M., et al. 2002, AcA, 52, 129

Zacharias, N., Finch, C., Girard, T., et al. 2010, AJ, 139, 2184 